\title{
Diagnosis of myocardial infarction in women: are we doing our best?
}

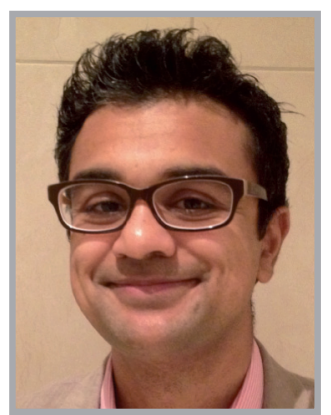

Q Can you tell us a little about your career background?

I am currently a clinical research fellow at the University of Edinburgh, British Heart Foundation Centre of Cardiovascular Science (UK). I completed my undergraduate and postgraduate training at the University of Edinburgh. I am now a Cardiology trainee in the southeast of Scotland.

Q You are a key author in a study to evaluate Abbott's (IL, USA) ARCHITECT STAT high-sensitivity troponin-I test. How did you get involved in this work?

The diagnostic criteria for myocardial infarction (MI) have not really changed over the last few decades. What has changed, however, is our ability to detect myocardial injury. Cardiac troponins have been used since the 1990s to detect injury to the heart. What has drastically changed over the last few years is the improvement in the clinical assays to measure troponin, such that we can now detect small concentrations of circulating troponin in a healthy reference population. Clearly, the advent of the new assays will significantly impact the diagnosis and subsequent treatment of MI, and we have been interested in evaluating the impact of these new assays on the clinical diagnoses of MI.

\section{Q Can you briefly explain the theory behind testing cardiac troponin levels in} patients with suspected MI?

Up until the 1990s, before troponin was introduced in clinical practice, the diagnosis of MI was based on clinical history, surface ECG and the presence of less sensitive biomarkers such as CK-MB.

Cardiac troponin is a three-piece regulatory protein that is present within the cytoskeletal structure of the cardiac myocyte. Over $90 \%$ of the cardiac troponin is bound within the cytoskeletal structure and only released into the bloodstream during injury to the cardiac myocyte.

Cardiac troponin, therefore, provided improved specificity for myocardial injury. This revolutionized the diagnosis of MI in the clinical arena. Over the last decade, the assays that measure cardiac troponin have become increasingly sensitive, changing the way we diagnose MI.

*Centre of Cardiovascular Sciences, Chancellors Building, Room SU 305, University of Edinburgh, Little France, Edinburgh, EH16 4SB, UK; anoop.shah@ed.ac.uk

\section{KEYWORDS}

- acute coronary syndrome

- troponin

“...we are now able to implement a sex-specific diagnostic threshold for cardiac troponin to detect myocardial injury [which] is likely to significantly improve the diagnostic accuracy for myocardial infarction in women."

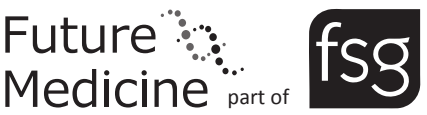




\section{“...we can now detect small concentrations of circulating troponin in a healthy reference population.”}

Q Why might this test particularly benefit women?

We have known for some time now that diagnosing MI in women is more challenging and this has previously been attributed to women having apparently less reliable presenting clinical features.

We also know that women generally have a smaller left ventricular mass compared with men. The ability for these new assays to detect cardiac troponin in the majority of a healthy reference population has shown significant gender differences in the normal reference range between men and women, with men generally having higher levels of circulating troponin. The fact that, with these new tests, we are now able to implement a sex-specific diagnostic threshold for cardiac troponin to detect myocardial injury is likely to significantly improve the diagnostic accuracy for MI in women.

\section{Q What are the aims of the study?}

The main aim of this study was to assess the potential impact of the diagnosis of MI using these high-sensitivity assays with genderspecific thresholds in a population of patients presenting with suspected acute coronary syndrome.

Q How many patients have been included in the study so far \& how many do you expect will be involved by the end date in 2016?

The current study was a prespecified substudy of the High-STEACS trial. In a single tertiary center study, we recruited just over 1000 consecutive men and women presenting with suspected acute coronary syndrome. Across the High-STEACS study, in ten participating centers within the central belt of Scotland, we aim to recruit 26,000 patients presenting to the emergency services with suspected acute coronary syndrome.

\section{Q What were the inclusion criteria for the study?}

The main inclusion criterion was that these patients were suspected to have an acute coronary syndrome and had a clinical troponin test requested. All consecutive patients presenting with suspected acute coronary syndrome were included in this substudy. Only the Abbott contemporary sensitive troponin-I assay was used to guide clinical care. At the same time, we measured cardiac troponin using the Abbott ARCHITECT high-sensitivity troponin-I assay. Two independent cardiologists then adjudicated the diagnosis for type I MI, initially using the contemporary sensitive assays followed by using the sex-specific and generic diagnostic thresholds on the high-sensitivity assay.

\section{Q How were the different diagnostic} thresholds calculated for men \& women? The diagnostic thresholds used were determined according to the Third Universal Definition of Myocardial Infarction published last year. The sex-specific thresholds at the 99th percentile using high-sensitivity assays were derived from a large healthy reference population with over 4000 samples.

\section{Q What was the end point of the study?}

The end point of the study was the diagnosis of a type I MI.

Q You have presented the preliminary results of this study at the European Society of Cardiology. What results have been observed so far?

We have noticed that using sex-specific thresholds on the high-sensitivity troponin-I assays makes little difference in the diagnosis of MI in men, but doubles the diagnosis of MI in women. Additionally, we noticed that women with elevated troponin levels above the 99th percentile, but below the current clinical diagnostic thresholds were at a high risk of recurrent $\mathrm{MI}$ and death at 6 months following the index event.

Q What do you expect to see from the final results of the trial after its completion in 2016 ?

We expect to see an increase in the diagnosis of MI. This is likely to be primarily due to an increase in the diagnosis rate in women. Whether identifying these patients and reclassifying them as MI improves outcomes will be evaluated by the trial.

\section{Q What do you think are the most} prominent themes/issues in women's cardiology?

Cardiology has advanced in multiple areas over the last few decades. However, it is still concerning that there remains sex-based 
discrepancies in the diagnosis and subsequent provision of evidence-based therapy in women with heart disease. One of the main issues is under-representation of women in large randomized trials.

Q Having attended the European Society of Cardiology this year, what do you think will be the most exciting advancements in cardiology in the next few years?

I think advancements in heart failure therapy and risk stratification in primary prevention are going to be two leading fields in cardiology in the next few years.

\section{Disclaimer}

The opinions expressed in this interview are those of the interviewee and do not necessarily reflect the views of Future Medicine Ltd.

Financial \& competing interests disclosure

A Shah has acted as a consultant and received honoraria from Abbott Diagnostics. The author has no other relevant affiliations or financial involvement with any organization or entity with a financial interest in or financial conflict with the subject matter or materials discussed in the manuscript apart from those disclosed.

No writing assistance was utilized in the production of this manuscript. 